\title{
Ulinastatin May Significantly Improve Postoperative Cognitive Function of Elderly Patients Undergoing Spinal Surgery by Reducing the Translocation of Lipopolysaccharide and Systemic Inflammation
}

\author{
Min Zhang ${ }^{1}$, Yan-Hua Zhang ${ }^{1}$, Hui-Qun Fu', Qing-Ming Zhang'2 and Tian-Long Wang ${ }^{1 *}$ \\ ${ }^{1}$ Department of Anesthesiology, Xuanwu Hospital, Capital Medical University, Beijing, China, ${ }^{2}$ Department of Orthopedics, \\ Xuanwu Hospital, Capital Medical University, Beijing, China
}

OPEN ACCESS

Edited by:

Patrizia Ballerini,

Università degli Studi G. d'Annunzio

Chieti e Pescara, Italy

Reviewed by:

Claudio Ferrante

Università degli Studi G. d'Annunzio

Chieti e Pescara, Italy

Antonio González-Pérez,

Centro Español de Investigación

Farmacoepidemiológica, Spain

*Correspondence:

Tian-Long Wang

w_tl5595@hotmail.com

Specialty section

This article was submitted to Inflammation Pharmacology,

a section of the journal

Frontiers in Pharmacology

Received: 09 April 2018

Accepted: 17 August 2018

Published: 09 October 2018

Citation:

Zhang $M$, Zhang $Y-H$, Fu H-Q,

Zhang Q-M and Wang T-L (2018) Ulinastatin May Significantly Improve

Postoperative Cognitive Function of Elderly Patients Undergoing Spinal

Surgery by Reducing

the Translocation

of Lipopolysaccharide and Systemic

Inflammation

Front. Pharmacol. 9:1007.

doi: 10.3389/fphar.2018.01007
Background: Studies have shown that perioperative inflammatory response is one of the important factors that caused postoperative cognitive dysfunction (POCD). Ulinastatin is a broad-spectrum protease inhibitor that inhibits inflammatory. We investigated the effects of ulinastatin on inflammatory response and early postoperative cognitive function in elderly patients undergoing spinal surgery.

Methods: This clinical trial was approved by the Xuanwu Hospital Ethical Committee (Registration number: ChiCTR-IPR-16008931). Sixty elderly patients undergoing elective spinal surgery with American Society of Anesthesiologists (ASA) status of I-II were randomized into ulinastatin and control groups; total intravenous anesthesia was performed. The elderly patients in ulinastatin group underwent intravenous infusion of ulinastatin 10,000 units/kg following anesthesia induction and before surgical incision, and 5000 units/kg on post-operative days 1 and 2. Cognitive function was determined with Montreal Cognitive Assessment (MOCA) test preoperatively and on post-operative day 7 by a neurologist. Serum lipopolysaccharide (LPS), interleukin-6 (IL-6), C-reactive protein (CRP), and matrix metalloprotease-9 (MMP-9) concentration levels were measured at baseline, the end of surgery, and on post-operative days 1 and 3 .

Results: All elderly patients completed the study. Ulinastatin infusion significantly reduced the incidence of $\mathrm{POCD}$ in elderly patients undergoing spine surgery (ulinastatin group $16 \%$ vs. control group 43\%, $\chi^{2}=5.079, P=0.024, P<0.05$ ). The elderly patients in ulinastatin group exhibited lower serum LPS, IL-6, CRP, and MMP-9 concentrations, as well as a shortened peak value duration, compared with those in the control group following surgery $(P<0.05)$.

Conclusion: Systemic inflammation and translocation of LPS were inhibited by the infusion of ulinastatin in elderly patients undergoing spinal surgery. The anti-inflammation intervention with ulinastatin can significantly improve the elderly patients' postoperative cognitive function.

Keywords: ulinastatin, elderly patients, spine surgery, lipopolysaccharide, systemic inflammation, POCD 


\section{INTRODUCTION}

The mechanism of postoperative cognitive dysfunction (POCD) in elderly individuals is related to excessive systemic inflammation caused by surgical trauma in elderly individuals, as well as an imbalance in the circulatory and central nervous system's immune responses (increased pro-inflammatory substances and reduced anti-inflammatory substances) (Nathan and Rodney, 2008; Hu et al., 2010). Perioperative alarms include the release of high mobility group protein (HMGB1), neutrophils, and monocyte cytoplasmic proteins (S100A8 and S100A9) from sterile wound tissue (Lu et al., 2015; Susana and Mervyn, 2016). These can stimulate the systemic inflammatory response, which plays a key role in the occurrence and development of POCD. Anti-inflammatory and anti-HMGB1 treatment can improve the occurrence of POCD (Rasmussen, 2006). Abdominal surgery can lead to destruction of the intestinal barrier, such that endotoxin (lipopolysaccharide, LPS) are absorbed into the blood where it can stimulate the systemic inflammatory response (Schietroma et al., 2013). However, the relationship between LPS levels, systemic inflammatory responses triggered by non-abdominal surgery, and POCD has not yet been elucidated.

Organic inflammatory response can promote the generation of MMP-9. MMP-9 can resolve and destroy the basement membrane of $\mathrm{BBB}$ (Blood Brain Barrier), may change the cerebral vascular permeability and cause the $\mathrm{BBB}$ impairment (Hu et al., 2012). BBB permeability changes can promote a large number of circulatory system inflammatory mediators around the central nervous system, a direct attack on neuronal cells or indirect activation of microglial cells, resulting in a series of physiological and pathological processes, and eventually resulting in abnormalities in nerve cells, as well as clinical manifestations of postoperative short-term cognitive decline (Bruno et al., 2009).

Ulinastatin is a urinary trypsin inhibitor (UTI) derived from human urine, which can inhibit enzyme activity and effectively reduce systemic inflammatory response (Atal and Atal, 2016). UTI, a serine protease inhibitor, has been widely used for patients with acute inflammatory disorders such as disseminated intravascular coagulation, shock, and pancreatitis (Inoue et al., 2005). Ulinastatin inhibits systemic inflammatory responses and reduces inflammatory factor expression and metalloproteinase activation. Concomitantly, ulinastatin can inhibit enzymatic activity and stabilize lysosomal membranes, such as matrix metalloprotease-9 (MMP-9) (Li et al., 2018), trypsin, granulocytic elastase, and cathepsin (Inoue et al., 2009; Lee et al., 2010; Fang et al., 2011). Through this mechanism, ulinastatin can protect intestinal barrier function. In addition, ulinastatin inhibits neutrophil and monocyte activation, preventing the release of inflammatory factors (Lee et al., 2010). However, whether ulinastatin can inhibit LPS and inflammatory factors triggered by non-abdominal surgery and improve cognitive function has not been elucidated. Therefore, the aim of this study was to determine the effects of ulinastatin on the inflammatory response and early POCD in elderly patients undergoing spinal surgery.

\section{MATERIALS AND METHODS}

\section{Patients and the Controls}

This study was a prospective, randomized, double-blind trial approved by the Xuanwu Hospital Ethical Committee (Registration number: ChiCTR-IPR-16008931); written informed consent was obtained from each patient. In this study, we enrolled 60 patients undergoing lumbar discectomy with general anesthesia and 30 age-matched control volunteers to examine the correlation between ulinastatin and serum lipopolysaccharide (LPS), MMP-9, interleukin-6 (IL-6), and C-reactive protein (CRP) levels with early POCD in patients.

We enrolled 60 patients older than 65 years (age $\leq 85$ years) with ASA status of I-II. Thirty community volunteers older than 65 years (age $\leq 85$ years) were examined to exclude the practice effect of repeated neuropsychological testing. Exclusion criteria were: Mini-mental State Examination (MMSE) score $\leq 23$, acute or chronic infectious diseases or trauma, tumor, taking anti-inflammatory drugs or immunosuppressants, $\mathrm{WBC} \geq 10000 \times 10^{9} / \mathrm{L}$ before surgery, a stroke in the prior 6 months or any other central nervous system diseases, lower digestive diseases, severe deafness or vision problems, illiteracy, and/or communication difficulties related to pronunciation or dialect and postoperative delirium, and refusal or unexpected discharge. We interviewed the patients on the day before surgery and collected baseline data, including age, sex, gender, body-mass index (BMI), past medical history, education history, and MMSE and Montreal Cognitive Assessment (MOCA) score.

Before the operation, patients were randomized into a control group (Group C) or a ulinastatin group (Group U). The randomization sequence without stratification was generated by a computer and sealed with consecutively numbered envelopes. The nurses were responsible for preparing ulinastatin or saline placebo with the same $100 \mathrm{ml}$ solution. Patients and investigators were all blinded to group allocation until the final statistical analysis was completed.

\section{Anesthesia}

All patients did not receive sedatives or anticholinergics before anesthesia. Induction of anesthesia was performed in all patients with sufentanil $0.3 \mu \mathrm{g} / \mathrm{kg}$, etomidate $0.2 \mathrm{mg} / \mathrm{kg}$, and rocuronium $0.8 \mathrm{mg} / \mathrm{kg}$. All patients were intubated and given $50 \%$ oxygen by pressure-controlled ventilation. Anesthesia was maintained by an infusion of propofol $3-5 \mathrm{mg} / \mathrm{kg} / \mathrm{h}$ and remifentanil $0.3 \mu \mathrm{g} / \mathrm{kg} / \mathrm{min}$ until the end of operation and sufentanil by intermittent injection (up to $0.5 \mu \mathrm{g} / \mathrm{kg}$ ). After the induction of anesthesia, ulinastatin 10,000 units/kg [diluted in normal saline to $100 \mathrm{ml}$ (treatment group)] or $100 \mathrm{ml}$ normal saline (control group) was administered intravenously over a period of $30 \mathrm{~min}$ before surgical incision and 5000 units/kg was administered after surgery on the 1 st and 2 nd days. All patients received intravenous patient-controlled analgesia postoperatively.

\section{Measurements and Laboratory Data}

To measure IL-6, MMP-9, CRP, and lipopolysaccharide (LPS), venous blood samples were obtained at four time points: before 
the induction of anesthesia (T0), at the end of operation (T1), and 24 and $72 \mathrm{~h}$ postoperatively (T2 and T3). Blood samples $(4 \mathrm{ml})$ were centrifuged at $3000 \mathrm{rcf}$ for $15 \mathrm{~min}$ at $-4^{\circ} \mathrm{C}$ and stored at $-70^{\circ} \mathrm{C}$. Cytokine levels of IL- 6 and MMP9 were measured by Enzyme-Linked Immunosorbent Assay (ELISA, R\&D Systems, Brea, CA, United States). The level of CRP was measured by immunoturbidimetry (Beckman Coulter, Indianapolis, IN, United States). The level of LPS was measured by ELISA.

\section{Neuropsychological Test}

We used the MMSE test to exclude patients and controls whose score was $\leq 23$; MOCA test was used to examine the cognitive decline level. We compared the MOCA score in community volunteers on the 1 st and 8 th days. We calculated the average changes to determine the practice effect (Table 1) and obtain the standard deviation (SD), on the 1st day. We compared the scores on the day before operation with the scores on post-operative day 7; we subtracted the practice effect and divided the community volunteers SD (from the 1st day), to obtain a $\mathrm{Z}$ score. A patient was considered to exhibit POCD if the $\mathrm{Z}$ score was $\geq 1.96 \mathrm{SD}$.

\section{Statistical Analysis}

Statistical analyses were performed by using SPSS-PC software 17.0. Sample capacity was calculated for a reduction of $30 \%$ in the incidence of POCD in the ulinastatin group, compared with the control group. On basis of $\alpha=0.05$ and $1-\beta=0.80,30$ patients were needed in each group. All data was normally distributed,

TABLE 1 | The practice effect of the community volunteers by MOCA test.

\begin{tabular}{lcc}
\hline The 1st day & The 7th day & Practice effect \\
\hline $26.23 \pm 1.64$ & $28.15 \pm 1.34$ & $1.92 \pm 1.19$
\end{tabular}

according to the Kolmogorov-Smirnov test. Numerical data (serum LPS, IL-6, CRP, and MMP-9 concentration) between the ulinastatin and control groups were analyzed using Student's $t$-test; intragroup numerical data were analyzed using repeatedmeasures analysis of variance. Nominal data were analyzed using $\chi^{2}$ test. All significant factors obtained in the $t$-test or $\chi^{2}$ test were modeled in the multivariable logistic regression analysis. $P<0.05$ was considered to indicate a significant difference.

\section{RESULTS}

A total of 103 patients older than 65 years (from 66 to 82 years old) were admitted. Forty-three patients were excluded, which was detailed in Figure 1. Finally, 60 patients were enrolled and randomized into this study.

There were no significant differences between the two groups in characteristics (age, gender, BMI, education, MMSE score, and operation time) (Table 2).

There were no significant differences between the two groups in these data. Numerical Value $=$ Mean \pm SD. BMI, body-mass index; MMSE, mini-medical state examination.

There were significant differences in the postoperative decline of the MOCA test. There was a lower incidence of POCD in the ulinastatin group $(5 / 30,16 \%)$ than in the control group $(13 / 30$, $43 \%), \chi^{2}=5.079, P=0.024, P<0.05$ (Table 3).

All postoperative data were adjusted to match perioperative data using hematocrit obtained by arterial blood gas analysis. In both control and ulinastatin groups, serum IL-6 concentrations increased at the end of surgery and on post-operative days 1 and 3 . However, the ulinastatin group showed lower serum IL6 concentrations than the control group on post-operative days 1 and $3(P<0.05)$ (Figure 2$)$.

In both control and ulinastatin groups, serum CRP concentrations increased on post-operative days 1 and 3 .

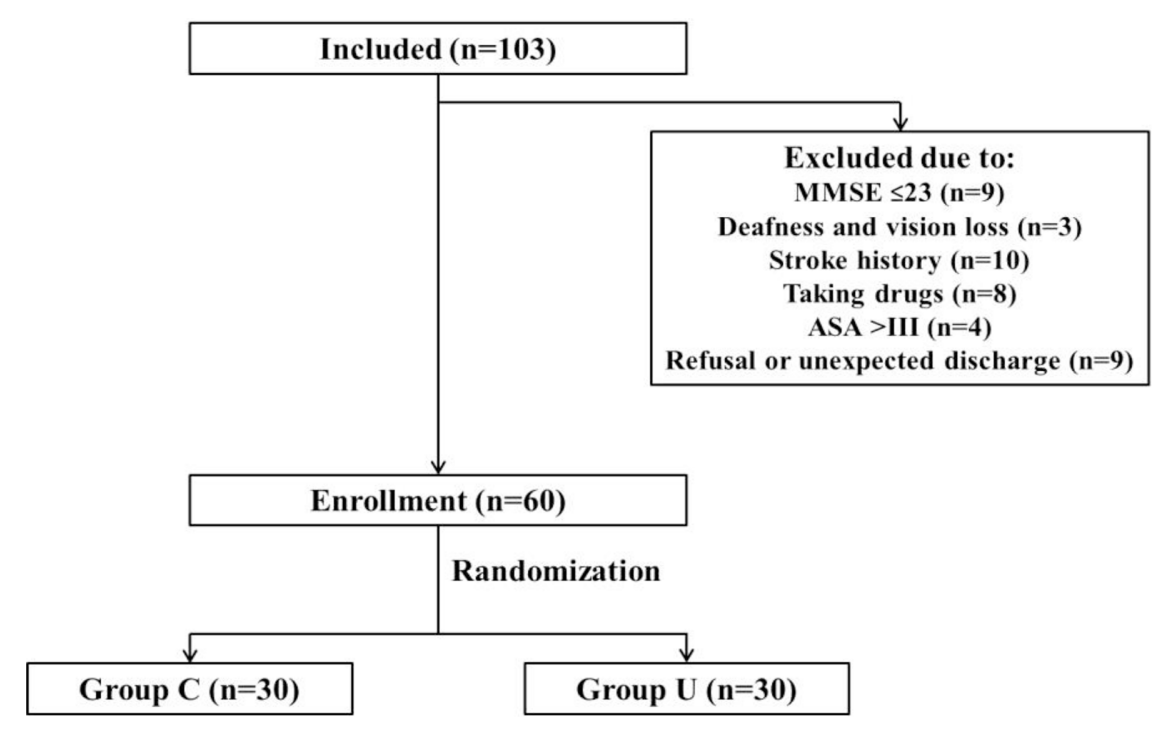

FIGURE 1 | The patient recruitment flow chat. 
TABLE 2 | Patient characteristics.

\begin{tabular}{lccc}
\hline & Group C $(\boldsymbol{n}=\mathbf{3 0})$ & Group U $(\boldsymbol{n}=\mathbf{3 0})$ & $\boldsymbol{P}$-values \\
\hline Gender (male/female) & $12 / 18$ & $14 / 16$ & 0.795 \\
Age (year) & $72.83 \pm 5.27$ & $71.26 \pm 4.95$ & 0.849 \\
BMl (kg/m²) & $25.36 \pm 0.75$ & $25.48 \pm 0.54$ & 0.905 \\
Education level (year) & $8.65 \pm 0.93$ & $9.08 \pm 0.37$ & 0.607 \\
Duration of surgery (min) & $228.53 \pm 14.51$ & $205.35 \pm 9.87$ & 0.089 \\
MMSE scores & $27.23 \pm 2.05$ & $26.36 \pm 2.64$ & 0.209 \\
\hline
\end{tabular}

TABLE 3 | The results of two groups' neuropsychological test.

\begin{tabular}{lccr}
\hline & 1st day & 7th day & POCD \\
\hline Group C & $25.20 \pm 2.44$ & $23.13 \pm 2.84$ & $13(n=30)$ \\
Group U & $24.32 \pm 3.31$ & $24.84 \pm 3.43$ & $5(n=30)$ \\
\hline
\end{tabular}

Cognitive change value $=$ Score the day before operation - Score $_{\text {the post-operation day } 7}$ practice effect. Values $=$ mean $\pm S D$.

$Z$ Score = Cognitive change value(patients)/SD(community volunteers).

$Z \geq 1.96$ was considered as POCD.

However, the ulinastatin group showed lower serum CRP concentrations than the control group on post-operative day 3 $(P<0.05)$ (Figure 3).

In both control and ulinastatin groups, serum MMP-9 concentrations increased at the end of surgery and on postoperative days 1 and 3. However, the ulinastatin group showed lower serum MMP-9 concentrations than the control group at the end of surgery and on post-operative days 1 and $3(P<0.05)$ (Figure 4).

In the control group, serum LPS concentration increased on post-operative day 3. In the ulinastatin group, serum LPS concentration increased at the end of surgery but decreased on post-operative days 1 and 3 . The ulinastatin group had lower serum LPS concentration than the control group on post-operative day $3(P<0.05)$ (Figure 5).

The crude OR (without covariates) is 0.262 (95\% CI:0.079$0.870, P<0.05$ ) and the adjusted OR (with all covariates) is 0.155 (95\% CI:0.028-0.855, $P<0.05$ ). The crude OR is some bigger than the adjusted OR, but statistical analyses all demonstrated that ulinastatin intervention was a protective factor for POCD (Table 4).

\section{DISCUSSION}

In this study, we found that patients in the ulinastatin group had lower levels of inflammatory cytokines (serum IL-6, CRP, MMP-9, and LPS) after the operation compared with the control group; further, there was a lower occurrence of POCD in the ulinastatin group, as assessed by neuropsychological testing. Logistic regression analysis demonstrated that ulinastatin intervention was a protective factor for POCD, which indicated that patients with ulinastatin intervention were not likely to experience POCD. We found that ulinastatin may inhibit the release of serum LPS, IL-6, CRP, and MMP-9, and may prevent the occurrence of POCD. The ulinastatin group had lower serum
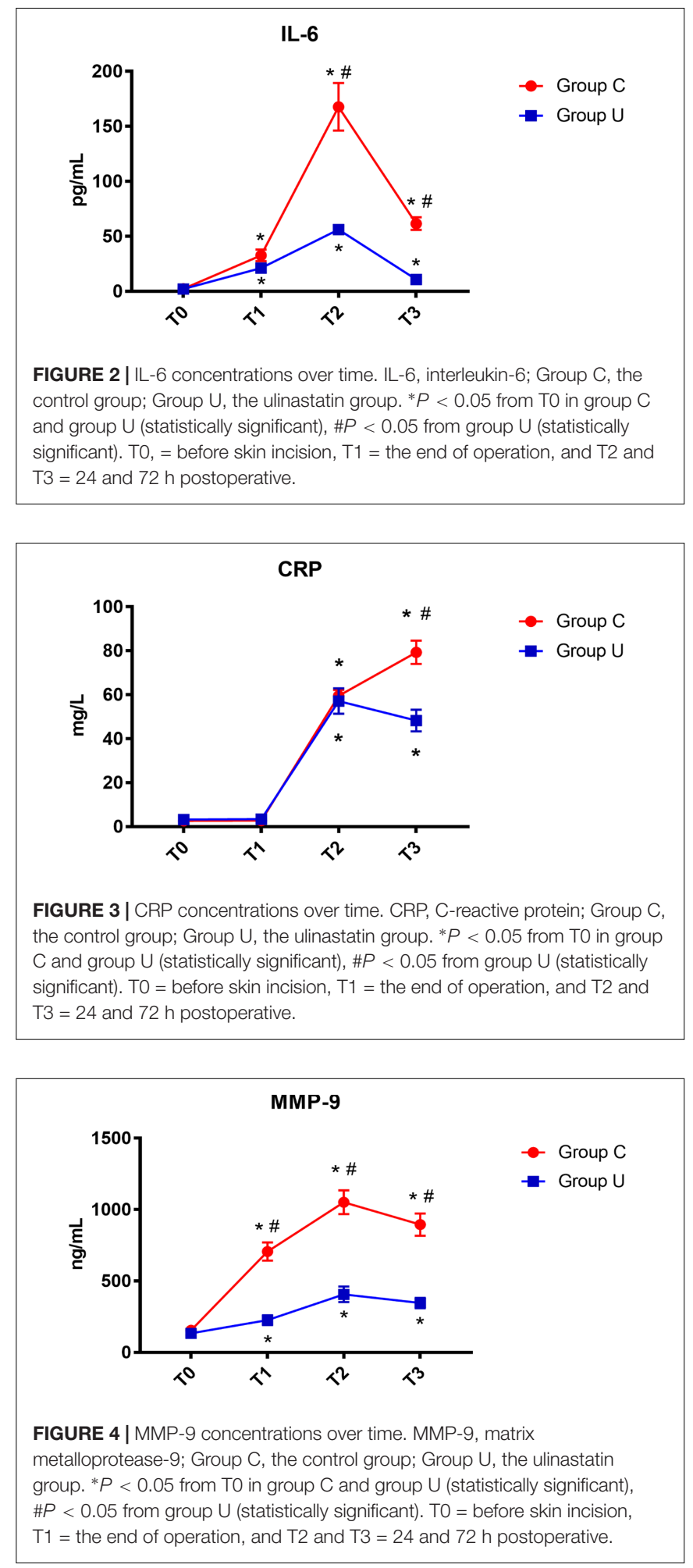

LPS concentration than the control group on post-operative day 3 .

Thereby, ulinastatin can reduce the destruction by inflammatory factors in the brain and improve neurological function in elderly patients. In addition, ulinastatin may utilize 


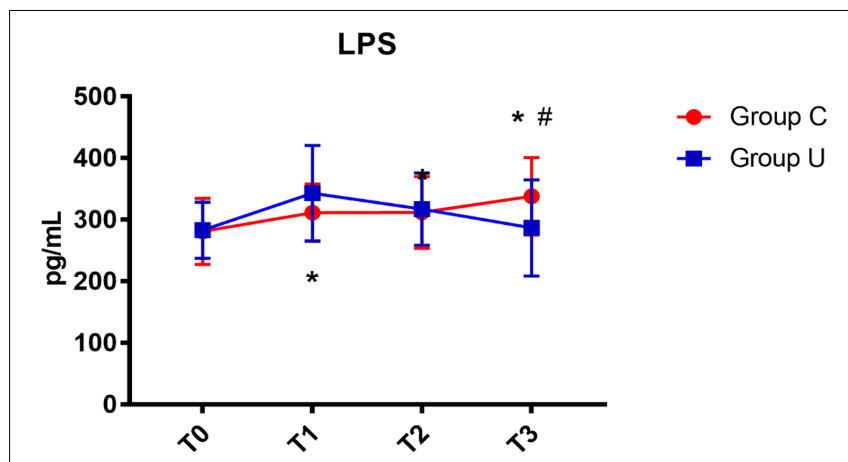

FIGURE 5 | LPS concentrations over time. LPS, lipopolysaccharide; Group C, the control group; Group $U$, the ulinastatin group. ${ }^{*} P<0.05$ from T0 in group $\mathrm{C}$ and group $\mathrm{U}$ (statistically significant), $\# P<0.05$ from group $U$ (statistically significant). $\mathrm{T0}=$ before skin incision, $\mathrm{T} 1=$ the end of operation, and $\mathrm{T} 2$ and $\mathrm{T} 3=24$ and $72 \mathrm{~h}$ postoperative.

TABLE 4 | Crude estimates and adjusted estimates of independent risk factors and protective factors for cognitive dysfunction.

\begin{tabular}{lcccccccc}
\hline & \multicolumn{3}{c}{ Crude estimates } & & \multicolumn{3}{c}{ Adjusted estimates } \\
\cline { 2 - 3 } Variations & $\mathbf{P}$ & OR & $\mathbf{9 5 \%} \mathbf{~ C l}$ & & $\mathbf{P}$ & $\mathbf{O R}$ & $\mathbf{9 5 \%} \mathbf{~ C l}$ \\
\hline Intervention & 0.024 & 0.262 & $0.079-0.870$ & & 0.032 & 0.155 & $0.028-0.855$ \\
Age & 0.150 & 1.086 & $0.971-1.216$ & & 0.241 & 1.076 & $0.952-1.216$ \\
Education & 0.182 & 0.858 & $0.686-1.074$ & & 0.191 & 0.855 & $0.675-1.082$ \\
Surgery & 0.514 & 1.010 & $0.979-1.043$ & & 0.233 & 0.973 & $0.931-1.018$ \\
duration & & & & & & & \\
\hline
\end{tabular}

an intestinal protection mechanism to protect brain function. Kohman et al. (2007) confirmed that intraperitoneal injection of LPS in rats induced toll-like receptor activation and increased inflammatory factors IL-1, IL-6, and tumor necrosis factor-alpha (TNF- $\alpha$ ), which eventually caused cognitive dysfunction. LPS, also called endotoxin, is the lipid-soluble outer-membrane component of Gram-negative bacteria. These bacteria are both pathogens and the commensal population in the human gut. The bacteria in the intestinal tract are a major source of LPS in humans. It is important that LPS may translocate from the intestine into the systemic circulation (Rhee, 2014). LPS protein complex with toll-like receptor-4 activates the cellular NF- $\kappa \mathrm{B}$ signaling pathway which, in turn, leads to production of proinflammatory cytokines throughout the body (Brun et al., 2007). LPS is a potent astrocyte activator and an inducer of brain inflammation, associated with proinflammatory cytokines in many experimental models both in vivo and in vitro ( $\mathrm{Li}$ et al., 2004; Weinstein et al., 2008). In our study, the ulinastatin group had a lower serum LPS concentration and a lower occurrence of POCD than the control group. This shows that ulinastatin inhibits LPS indirectly by inhibiting the systemic inflammatory response, which can damage the intestinal barrier, and is associated with the occurrence of intestinal bacterial migration. Intestinal ischemia caused by surgical stress easily leads to decreased intestinal barrier function and intestinal endotoxin transfer to the blood, which significantly increases blood LPS levels and induces systemic inflammatory response (Yeh et al., 2012). In our study, we tracked the level of LPS within $72 \mathrm{~h}$ after surgery and found that the level of LPS in elderly patients was significantly higher than before surgery. This shows that our perioperative inflammatory response is not only derived from the surgical trauma, but also from the fact that we do not have effective stress control, which resulted in an imbalance of intestinal oxygen supply and demand, endotoxin migration, and triggering of a systemic inflammatory response.

In our study, we administered ulinastatin 10,000 U/kg before anesthesia and 5,000 U/kg on the post-operative 1st and 2nd days because the half-life time of ulinastatin is about $40 \mathrm{~min}$ in healthy volunteers (Oh et al., 2012). Our study demonstrated that the level of serum CRP in the control group increased on post-operative days 1 and 3, but the level of serum CRP in the ulinastatin group increased on post-operative day 1 and decreased on post-operative day 3. It is now believed that the key inflammatory response promoter is TNF- $\alpha$, but the concentration of CRP increases significantly with the degree of surgical trauma after surgery and will continue to 24-72 h after surgery (Zhang et al., 2015). In small surgery, CRP lasted 24-48 $\mathrm{h}$ with a peak value of $40 \mathrm{mg} / \mathrm{L}$; during nephrectomy, the inflammatory response time increased from 48 to $72 \mathrm{~h}$ with the peak value reaching $128 \mathrm{mg} / \mathrm{L}$, which was double that of $40 \mathrm{mg} / \mathrm{L}$ (Watt et al., 2015).

Our data demonstrated that the concentration of serum IL-6 in the control group and the ulinastatin group increased at the end of surgery and on post-operative days 1 and 3. However, the ulinastatin group showed lower serum IL- 6 concentrations than the control group on post-operative days 1 and $3(p<0.05)$. This suggested that ulinastatin may inhibit the release of IL-6. Serum IL-6 is a sensitive marker of tissue damage and is also directly related to the duration of surgery (Maier et al., 2007). There was a significant positive correlation between the serum MMP-9 level and the POCD severity. Organic inflammatory response can promote the generation of MMP-9. MMP-9 can resolve and destroy the basement membrane of $\mathrm{BBB}$ (Blood Brain Barrier), may change the cerebral vascular permeability and cause the BBB impairment (Hu et al., 2012). In our study, the concentration of MMP-9 increased quickly at the end of operation and was higher in control group than in the ulinastatin group at 24 and $72 \mathrm{~h}$ postoperation. This suggests that the central nervous system or the integrity of BBB may be some degree damaged in short-term, especially in the control group.

Ulinastatin is a protease inhibitor isolated from the urine of healthy adult males. It is not easy to be allergic and safe to use. It can reduce the body's inflammatory response through various ways such as antioxidant reaction, anti-proteolysis, and inhibition of inflammatory mediator release. Lv et al. (2016) reported that Ulinastatin administration was effective in treating early POCD (post-operative days 3 and 7) and reducing IL-6 and $S 100 \beta$ concentrations within 2 days after operations. Lili et al. (2013) reported that the ulinastatin group had a lower incidence of POCD than the control group (2.5 versus $27.5 \%$, $p<0.05)$ and the ulinastatin group had lower serum S100 $\beta$ protein and IL- 6 concentrations than those in the control group. 
Our observed effect (ulinastatin group 16\% vs. control group $43 \%$ ) is remarkable. The intervention reduced the incidence of POCD by almost two thirds. Ulinastatin has a broader research perspective and larger-scale studies are urgently needed in the future.

Postoperative cognitive disturbance, including postoperative delirium (POD) and POCD, is a common complication of perioperative neurocognition in elderly patients undergoing surgery. POD is characterized by inattention, disorganized thinking, and an altered level of consciousness. Generally, POD mostly occurs in the first 3 days following surgery (Erdem et al., 2016). POCD is characterized by cognitive impairment of memory, comprehension and attention. POCD usually occurs in the days to weeks following surgery (Rudolph et al., 2008). In our study, we used confusion assessment method (CAM) to diagnose POD on post-operative days 3 and 7. If Patients are diagnosed as delirium, they are rejected from the study.

This study has some limitations. Firstly, the follow-up study time was short. Rundshagen (2014) reported that $40 \%$ of patients (age $>60$ ) have POCD after operation and after 3 months later the incidence of POCD is $10 \%$. In our study, we had only performed the MOCA test at the day before operation and the post-operative 7 days. We should go on with the research on the base of the present result. Secondly, we should not preclude an effect of ulinastatin on TNF and other early markers of damage. Thirdly, the MOCA test is easier than the ISPOCD test studies, but is still some difficult for some patients who had lower education degrees.

In conclusion, POCD is very common in elderly patients undergoing surgical operations and its mechanism is very complex. In our study, owing to elderly gut fragility, LPS

\section{REFERENCES}

Atal, S. S., and Atal, S. (2016). Ulinastatin - a newer potential therapeutic option for multiple organ dysfunction syndrome. J. Basic Clin. Physiol. Pharmacol. 2, 91-99. doi: 10.1515/jbcpp-2015-0003

Brun, P., Castagliuolo, I., Di Leo, V., Buda, A., Pinzani, M., Palù, G., et al. (2007). Increased intestinal permeability in obese mice: new evidence in the pathogenesis of nonalcoholic steatohepatitis. Am. J. Physiol. Gastrointest. Liver Physiol. 292, 518-525. doi: 10.1152/ajpgi.00024.2006

Bruno, M. A., Mufson, E. J., Wuu, J., and Cuello, A. C. (2009). Increased matrix metalloproteinase -9 activity in mild cognitive impairment. J. Neuropathol. Exp. Neurol. 68, 1309-1318. doi: 10.1097/NEN.0b013e3181c22569

Erdem, A. F., Kayabasoglu, G., Tas, Tuna A, Palabiyik, O., Tomak, Y., and Beyaz, S. G. (2016). Effect of controlled hypotension on regional cerebral oxygen saturation during rhinoplasty: a prospective study. J. Clin. Monit. Comput. 30, 655-660. doi: 10.1007/s10877-015-9768-6

Fang, Y., Xu, P., Gu, C., Wang, Y., Fu, X. J., Yu, W. R., et al. (2011). Ulinastatin improves pulmonary function in severe burn-induced acute lung injury by attenuating inflammatory response. J. Trauma 71, 1297-1304. doi: 10.1097/TA. 0b013e3182127d 48

Hu, X., Zhang, M., Leak, R. K., Gan, Y., Li, P., Gao, Y., et al. (2012). Delivery of neurotherapeutics across the blood brain barrier in stroke. Curr. Pharm. 18, 3704-3720. doi: 10.2174/138161212802002715

Hu, Z., Ou, Y., Duan, K., and Jiang, X. (2010). Inflammation: a bridge between postoperative cognitive dysfunction and Alzheimer's disease. Med. Hypotheses 74, 722-724. doi: 10.1016/j.mehy.2009.10.040

Inoue, K., Takano, H., Sato, H., Yanagisawa, R., and Yoshikawa, T. (2009). Protective role of urinary trypsin inhibitor in lung expression of proinflammatory cytokines accompanied by lethal liver injury in is demonstrated to be a marker for cognitive dysfunction. Ulinastatin can inhibit the release of serum LPS, IL-6, CRP, and MMP-9, and prevent the occurrence of POCD. Further ulinastatin clinical studies about the effect on POCD in people are needed.

\section{AUTHOR CONTRIBUTIONS}

MZ was the first author of this article, responsible for the design and implementation of this project, data collection, data statistics, and article writing. Y-HZ was responsible for the design and data collection in the project. H-QF was responsible for the article modification. Q-MZ was responsible for the postoperative patient data collection. T-LW was the corresponding author of this article in charge of the project, responsible for the expenses of the project, design, writing, and checking the article.

\section{FUNDING}

This study was supported by Beijing Municipal Administration of Hospitals Clinical Medicine Development of Special Funding Support (Nos. ZYLX201818 and ZYLX201706), Beijing Municipal Administration of Hospital Ascent Plan (No. DFL20150802), National Clinical Research Center for Geriatric Disorders, Beijing, China, Beijing Municipal Commission of Health and Family Planning (Nos. PXM2018_026283_000002 and PXM2017_026283_000002), and Xuanwu Hospital of Capital Medical University, Beijing Institute of Geriatrics, Beijing, China.

mice. Immunopharmacol. Immunotoxicol. 31, 446-450. doi: 10.1080/ 08923970902769646

Inoue, K., Takano, H., Yanagisawa, R., Sakurai, M., Shimada, A., Yoshino, S., et al. (2005). Protective role of urinary trypsin inhibitor in acute lung injury induced by lipopolysaccharide. Exp. Biol. Med. 4, 281-287. doi: 10.1177/ 153537020523000408

Kohman, R. A., Tarr, A. J., Byler, S. L., and Boehm, G. W. (2007). Age increases vulnerability to bacterial endotoxin-induced behavioral decrements. Physiol. Behav. 91, 561-565. doi: 10.1016/j.physbeh.2007.03.032

Lee, J. Y., Lee, J. Y., Chon, J. Y., Moon, H. S., and Hong, S. J. (2010). The effect of ulinastatin on hemostasis in major orthopedic surgery. Korean J. Anesthesiol. 58, 25-30. doi: 10.4097/kjae.2010.58.1.25

Li, G., Sun, S., Cao, X., Zhong, J., and Tong, E. (2004). LPS-induced degeneration of dopaminergic neurons of Substantia nigra in rats. J. Huazhong Univ. Sci. Technolog. Med. Sci. 24, 83-86.

Li, X. F., Zhang, X. J., Zhang, C., Wang, L. N., Li, Y. R., Zhang, Y., et al. (2018). Ulinastatin protects brain against cerebral ischemia/reperfusion injury through inhibiting MMP-9 and alleviating loss of ZO-1 and occludin proteins in mice. Exp. Neurol. 302, 68-74. doi: 10.1016/j.expneurol.2017.12.016

Lili, X., Zhiyong, H., and Jianjun, S. (2013). A preliminary study of the effects of ulinastatin on early postoperative cognition function in patients undergoing abdominal surgery. Neurosci. Lett. 541, 15-19. doi: 10.1016/j.neulet.2013. 02.008

Lu, S. M., Yu, C. J., Liu, Y. H., Dong, H. Q., Zhang, X., Zhang, S. S., et al. (2015). S100A8 contributes to postoperative cognitive dysfunction in mice undergoing tibial fracture surgery by activating the TLR4/MyD88 pathway. Brain Behav. Immun. 44, 221-234. doi: 10.1016/j.bbi.2014.10.011

Lv, Z. T., Huang, J. M., Zhang, J. M., Zhang, J. M., Guo, J. F., and Chen, A. M. (2016). Effect of ulinastatin in the treatment of postperative cognitive 
dysfunction: review of current literature. Biomed Res. Int. 2016:2571080. doi: $10.1155 / 2016 / 2571080$

Maier, B., Lefering, R., Lehnert, M., Laurer, H. L., Steudel, W. I., Neugebauer, E. A., et al. (2007). Early versus late onset of multiple organ failure is associated with differing patterns of plasma cytokine biomarker expression and outcome after severe trauma. Shock 28, 668-674. doi: 10.1097/shk.0b013e318123e64e

Nathan, L., and Rodney, W. (2008). Neuroinflammation associated with aging sensitized the brain to the effects of infection or stress. Neuroimmunomodulation 15, 323-330. doi: 10.1159/000156474

Oh, S. Y., Kim, J. C., Choi, Y. S., Lee, W. K., Lee, Y. K., and Kwak, Y. L. (2012). Effects of ulinastatin treatment on myocardial and renal injury in patients undergoing aortic valve replacement with cardiopulmonary bypass. Korean J. Anesthesiol. 62, 148-153. doi: 10.4097/kjae.2012.62.2.148

Rasmussen, L. S. (2006). Postoperative cognitive dysfunction: incidence and prevention. Best Pract. Res. Clin. Anaesthesiol. 20, 315-330.

Rhee, S. H. (2014). Lipopolysaccharide: basic biochemistry, intracellular signaling, and physiological impacts in the gut. Intest. Res. 12, 90-95. doi: 10.5217/ir.2014. 12.2.90

Rudolph, J. L., Marcantonio, E. R., Culley, D. J., Silverstein, J. H., Rasmussen, L. S., Crosby, G. J., et al. (2008). Delirium is associated with early postoperative cognitive dysfunction. Anaesthesia 63, 941-947. doi: 10.1111/j.1365-2044.2008. 05523.x

Rundshagen, I. (2014). Postoperative cognitive dysfunction. Dtsch. Arztebl. Int. 111, 119-125. doi: 10.3238/arztebl.2014.0119

Schietroma, M., Pessia, B., Carlei, F., Cecilia, E. M., and Amicucci, G. (2013). Intestinal permeability, systemic endotoxemia, and bacterial translocation after open or laparoscopic resection for colon cancer: a prospective randomized study. Int. J. Colorectal. Dis. 28, 1651-1660. doi: 10.1007/s00384-013-1751-4

Susana, V., and Mervyn, M. (2016). Initiating mechanisms of surgery-induced memory decline: the Role of HMGB1. J. Clin. Cell. Immunol. 7:481. doi: 10. 4172/2155-9899
Watt, D. G., Horgan, P. G., and McMillan, D. C. (2015). Routine clinical markers of the magnitude of the systemic inflammatory response after elective operation: a systematic review. Sugery 157, 362-380. doi: 10.1016/j.surg.2014. 09.009

Weinstein, J. R., Swarts, S., Bishop, C., Hanisch, U. K., and Moller, T. (2008). Lipopolysaccharide is a frequent and significant contaminant in microglia-activating factors. Glia 56, 16-26. doi: 10.1002/glia. 20585

Yeh, Y. C., Sun, W. Z., Ko, W. J., Chan, W. S., Fan, S. Z., Tsai, J. C., et al. (2012). Dexmedetomidine prevents alterations of intestinal microcirculation that are induced by surgical stress and pain in a novel rat model. Anesth. Analg. 115, 46-53. doi: 10.1213/ANE.0b013e3182 53631c

Zhang, Y. H., Guo, X. H., Zhang, Q. M., Yan, G. T., and Wang, T. L. (2015). Serum CRP and urinary trypsin inhibitor implicate postoperative cognitive dysfunction especially in elderly patients. Int. J. Neurosci. 125, 501-506. doi: $10.3109 / 00207454.2014 .949341$

Conflict of Interest Statement: The authors declare that the research was conducted in the absence of any commercial or financial relationships that could be construed as a potential conflict of interest.

The reviewer CF and handling Editor declared their shared affiliation.

Copyright (c) 2018 Zhang, Zhang, Fu, Zhang and Wang. This is an open-access article distributed under the terms of the Creative Commons Attribution License (CC BY). The use, distribution or reproduction in other forums is permitted, provided the original author(s) and the copyright owner(s) are credited and that the original publication in this journal is cited, in accordance with accepted academic practice. No use, distribution or reproduction is permitted which does not comply with these terms. 